\title{
Simulations of supersymmetric Yang-Mills theory
}

\author{
K. Demmouche ${ }^{a}$, F. Farchioni ${ }^{a}$, A. Ferling ${ }^{a}$, I. Montvay ${ }^{b}$, G. Münster ${ }^{* a}$, E.E. Scholz ${ }^{c}$, \\ J. Wuilloud ${ }^{a}$ \\ ${ }^{a}$ Universität Münster, Wilhelm-Klemm-Strasse 9, D-48149 Münster, Germany \\ ${ }^{b}$ Deutsches Elektronen-Synchrotron DESY, Notkestr. 85, D-22603 Hamburg, Germany \\ ${ }^{c}$ Fermi National Accelerator Laboratory, Batavia, IL 60510, USA \\ E-mail: munsteg@uni-muenster.de
}

\begin{abstract}
Results of a numerical simulation concerning the low-lying spectrum of four-dimensional $\mathscr{N}=1$ SU(2) Supersymmetric Yang-Mills (SYM) theory on the lattice with light dynamical gluinos are reported. We use the tree-level Symanzik improved gauge action and Wilson fermions with stout smearing of the gauge links in the Wilson-Dirac operator. The configurations are produced with the Two-Step Polynomial Hybrid Monte Carlo (TS-PHMC) algorithm. We performed simulations on lattices up to a size of $24^{3} \cdot 48$ at $\beta=1.6$. Using QCD units with the Sommer scale being set to $r_{0}=0.5 \mathrm{fm}$, the lattice spacing is about $a \simeq 0.09 \mathrm{fm}$, and the spatial extent of the lattice corresponds to $2.1 \mathrm{fm}$ to control finite size effects. At the lightest simulated gluino mass our results indicate a mass for the lightest gluino-glue bound state, which is considerably heavier than the values obtained for its possible superpartners. Whether supermultiplets are formed remains to be studied in upcoming simulations.
\end{abstract}

The XXVII International Symposium on Lattice Field Theory

July 26-31, 2009

Peking University, Beijing, China

${ }^{*}$ Speaker. 


\section{Introduction}

Supersymmetric theories have received increasing interest in elementary particle physics. The supersymmetric extension of the Standard Model with $\mathscr{N}=1$ supercharge and other models beyond the Standard Model have supersymmetry (SUSY) as an essential ingredient.

The minimal supersymmetric extension of the $\mathrm{SU}\left(N_{c}\right)$ gauge theory describing self-interactions of gauge fields $A_{\mu}^{a}$, corresponding to the gluons ( $g$ ), is given by the $\mathscr{N}=1$ Supersymmetric YangMills (SYM) theory. The supersymmetric partners of the gluons are described by spin-1/2 Majorana fermion fields $\lambda_{a}\left(a=1 \ldots N_{c}^{2}-1\right)$, the gluinos $(\tilde{g})$. Compatibility of SUSY with gauge invariance requires that the gluinos transform in the adjoint representation of the gauge group. This theory describes the interactions between gluons and gluinos. The Lagrangian of SYM theory in the continuum, including a SUSY breaking mass term, reads

$$
\mathscr{L}_{S Y M}=-\frac{1}{4} F_{\mu \nu}^{a} F^{a \mu v}+\frac{\mathrm{i}}{2} \bar{\lambda}^{a}(x) \gamma^{\mu}\left(\mathscr{D}_{\mu} \lambda(x)\right)^{a}-\frac{m_{\tilde{g}}}{2} \bar{\lambda}^{a} \lambda^{a} .
$$

The mass term here introduces a soft breaking of supersymmetry.

In the low-energy regime, where the interactions become strong, arguments based on the lowenergy effective Lagrangian approach [1,2] predict the occurrence of non-perturbative dynamics like confinement and spontaneous chiral symmetry breaking in SUSY gauge theories. Confinement is realised by colourless bound states. In the case where the last term in Eq. (1.1) is switched off $\left(m_{\tilde{g}}=0\right)$, the anomalous global chiral symmetry $\mathrm{U}(1)_{\lambda}$ is present. The anomaly does not break the global chiral symmetry completely and a discrete subgroup $Z_{2 N_{c}}$ remains. As in the case of QCD, the discrete chiral symmetry is expected to be spontaneously broken to $Z_{2}$ by the nonvanishing value of the gluino condensate $\langle\bar{\lambda} \lambda\rangle$. The consequence of this spontaneous breaking is the existence of $N_{c}$ degenerate ground states with different orientations of the gluino condensate.

Another interesting aspect of SYM is its equivalence to QCD with a single quark flavour $\left(N_{f}=1 \mathrm{QCD}\right)$ in the limit of a large number of colours $\left(N_{c} \rightarrow \infty\right)$, where the Majorana spinor is replaced by the single Dirac spinor [3]. The latter model is also object of investigation by our collaboration [4].

A lattice formulation of SYM suitable for numerical simulations, employing Wilson fermions, has been proposed by Curci and Veneziano [5]. First non-perturbative investigations of SYM on the lattice using this formulation have been performed by the DESY-Münster-Roma collaboration; for a review see Ref. [6] and references [7, 8, 9, 10, 11]. SUSY is broken explicitly by the lattice discretisation. In the Wilson approach the mass term and the Wilson-term break both chirality and SUSY. Both symmetries are expected to be recovered in the continuum limit by tuning the relevant bare mass term to its critical value corresponding to a massless gluino $\left(m_{\tilde{g}}=0\right)$, and the gauge coupling towards zero.

In recent years, simulations of $\mathscr{N}=1 \mathrm{SYM}$ on the lattice using Ginsparg-Wilson fermions with good chiral properties, such as domain wall fermions, have been initiated [12, 13, 14]. For large lattice volumes and small lattice spacings these formulations require, however, a significantly larger amount of computing resources than the Wilson formulation. The gain of no need for tuning the position of the zero gluino mass point does not compensate by far the advantage of Wilson fermions. 
Here we report on recent work continuing the project of the DESY-Münster collaboration for the simulation of $\mathscr{N}=1 \mathrm{SU}(2) \mathrm{SYM}$. We present results in the light of the newly used TS-PHMC algorithm and improved actions.

The most important characteristics of the theory is the mass spectrum of bound states, for which the low-energy effective theories predict a reorganisation of the masses in two massive WessZumino supermultiplets at the SUSY point [1,2], where the soft breaking vanishes. The introduction of a small gluino mass removes the mass degeneracy between the supermultiplet members.

\section{Lattice formulation of $\mathscr{N}=1 \mathrm{SYM}$ theory}

For the gauge fields we employ the tree-level improved Symanzik (tlSym) action. The gluinos are represented by Majorana fermions $\lambda^{a}$ in the adjoint representation. The fermion part of the Curci-Veneziano action, describing the gluinos, is given by

$$
S_{\tilde{g}}^{W}=\frac{1}{2} \sum_{x} \bar{\lambda}(x) \lambda(x)-\frac{\kappa}{2} \sum_{x} \sum_{\mu}\left[\bar{\lambda}(x+\hat{\mu}) V_{\mu}(x)\left(1+\gamma_{\mu}\right) \lambda(x)+\bar{\lambda}(x) V_{\mu}^{T}(x)\left(1-\gamma_{\mu}\right) \lambda(x+\hat{\mu})\right],
$$

where $\kappa$ is the bare hopping parameter which encodes the bare gluino mass $\kappa=\left(2 m_{\tilde{g}, 0}+8\right)^{-1}$. The real orthogonal matrices $V_{\mu}(x)$ are the gauge links in the adjoint representation.

The links $U_{x, \mu}$ in the fermion action can be replaced by stout-smeared links [15]. This has the advantage that short range topological defects of the gauge field and the corresponding small eigenvalues of the fermion matrix are removed. We prefer to keep the action well localised and hence only perform a single stout-smearing step.

Similarly to QCD, the mass term proportional to $m_{\tilde{g}, 0}$ breaks chirality. In the present case it also breaks the supersymmetry. A massless gluino, $m_{\tilde{g}}=0$, is obtained by tuning the relevant bare mass term to its critical value ( $m_{0} \rightarrow m_{0 c r}$ ) or equivalently $\kappa \rightarrow \kappa_{c r}$.

The fermion action can be rewritten in term of an antisymmetric matrix $M=\mathscr{C} Q$, where $Q$ is the non-hermitian fermion matrix or lattice Wilson-Dirac operator for Dirac fermions in the adjoint representation, and $\mathscr{C}$ is the charge conjugation matrix in the spinorial representation. Integration of the fermionic variables yields the Pfaffian of $M$, whose absolute value equals the square root of the fermion determinant. Effectively, this corresponds to a flavour number $N_{f}=\frac{1}{2}$. In the Wilson setup the Pfaffian can become negative even for positive gluino masses.

In our numerical simulations we include the dynamics of the gluino by the Two-Step Polynomial Hybrid Monte Carlo (TS-PHMC) [16] algorithm with flavour number $N_{f}=\frac{1}{2}$. This has the consequence that only the absolute value of the Pfaffian is taken into account in the updating of the gauge field configuration. The sign of the Pfaffian has to be included in a reweighting step when calculating expectation values. It can be shown that the sign of the Pfaffian is equal to the sign of the product of half of the doubly degenerate negative real eigenvalues of $Q$. For positive gluino masses sufficiently far away from zero a negative sign of the Pfaffian rarely occurs in the updating sequence and therefore a sign problem does not show up.

The TS-PHMC algorithm turned out to be very efficient in producing short autocorrelations among the gauge configurations. For instance, in the stout-smeared runs on a $24^{3} \cdot 48$ lattice the integrated autocorrelation of the average plaquette (which belongs to the worst quantities from the point of view of autocorrelations) did always satisfy $\tau_{\text {int }}^{\text {plaq }}<10$. 
The values of the gauge coupling parameter $\beta$ can be fixed by investigating the static potential of an external fundamental charge and extracting the Sommer scale parameter $r_{0} / a$ [17]. Note that in analogy with QCD, we set the value of $r_{0}$ by definition to $r_{0}=0.5 \mathrm{fm}$. In this way we can use familiar QCD units for physical dimensionful quantities.

The TS-PHMC runs were done on $16^{3} \cdot 32$ and $24^{3} \cdot 48$ lattices at $\beta=1.6$ at various values of the hopping parameter $\kappa$. On the $24^{3} .48$ lattice four points have been simulated with stout-links. The lattice spacing amounts to $a \simeq 0.09 \mathrm{fm}$. The lattice extension $L \simeq 2.1 \mathrm{fm}$ is expected to be large enough to allow control over finite volume effects on the bound state masses.

The dimensionless quantity $M_{r} \equiv\left(r_{0} m_{\pi}\right)^{2}$, where $m_{\pi}$ is the pion mass in the corresponding theory with two Dirac fermions in the adjoint representation, is expected to be proportional to the gluino mass (see below), and can be considered to be a measure of it.

An issue in lattice simulation is the lightness of the dynamical quarks which leads to slowing down of the update algorithms. With the new TS-PHMC algorithm the lightest adjoint pion mass in our simulations was about $440 \mathrm{MeV}$. Simulations for smaller gluino masses and/or finer lattice spacings are going on presently.

\section{Low-lying bound state spectrum}

For the investigation of the low-lying bound state spectrum we concentrate on the projecting operators employed for the construction of the low-energy Lagrangians of [1] and [2]. These are expected to dominate the dynamics of SYM at low energies. Previous experience on the field can be found in [7] and [9]. We investigate spin-0 gluino-gluino bilinear operators (adjoint mesons), a spin-1/2 mixed gluino-glue operator and spin-0 glueball operators. In some cases smearing techniques such as APE [18] and Jacobi smearing [19] are applied in order to increase the overlap of the lattice operator with the low-lying bound state.

The adjoint mesons are colourless composite states of two gluinos with spin-parity $0^{+}$and $0^{-}$. In analogy to flavour singlet states in QCD we denote the former $a-\eta^{\prime}$ and the latter $a$ - $f_{0}$, where the prefix $a$ stays for "adjoint". The associated projecting operators are the gluino bilinear operators $\mathscr{O}_{\text {mes }}=\bar{\lambda} \Gamma \lambda$ where $\Gamma=\gamma_{5}, 1$ respectively.

The resulting propagator consists of connected and disconnected contributions. The exponential decay of the connected term defines the adjoint pion mass $m_{a-\pi}$. This quantity, even if not associated to a physical state of SYM, can be used to characterise the gluino mass. Indeed, according to arguments involving the OZI-approximation of SYM, the adjoint pion mass is expected to vanish for a massless gluino and the behaviour $m_{a-\pi}^{2} \propto m_{\tilde{g}}$ can be assumed for light gluinos [1,9].

For the positive parity glueball $0^{+}$we adopted the simplest interpolating operator built from space-like plaquettes. In order to improve the signal we applied APE smearing with the variational method [20].

Also here, as for the scalar $a-f_{0}$, the gauge samples generated with stout links turn out to generally give better results for the glueball masses.

The gluino-glueballs $(\tilde{g}-g)$ are spin- $\frac{1}{2}$ colour singlet states of a gluon and a gluino. They are supposed to complete the Wess-Zumino supermultiplet of the adjoint mesons [1]. We adopt for this state the lattice version of the gluino-glue operator $\operatorname{Tr}_{c}[F \sigma \lambda]$ [1], where the field-strength tensor $F_{\mu v}(x)$ is replaced by the clover-plaquette operator $P_{\mu v}(x)[21,9]$. We apply APE smearing for the 
links and Jacobi smearing for the fermion fields in order to optimise the signal-to-noise ratio and to obtain an earlier plateau in the effective mass.

A vanishing gluino mass is a prerequisite for supersymmetry in the continuum limit. Therefore the point corresponding to a massless gluino is of high interest in lattice simulations of SYM. With Wilson fermions this point must be located by tuning the hopping parameter. The subtracted gluino mass can be determined in a direct way from the study of lattice SUSY Ward-Identities (WIs) [21] and, in an indirect way, from the vanishing of the adjoint pion mass. Indeed, as mentioned above, the pion mass squared $\left(a m_{\pi}\right)^{2}$ is expected to vanish linearly with the (renormalised) gluino mass. Both the WIs and adjoint pion mass methods give consistent estimates of the critical hopping parameter $\kappa_{c r}$ corresponding to vanishing gluino mass.

The masses of the lightest bound states of low-energy $\mathscr{N}=1$ SYM determined in this work are shown in Fig. 1. The masses of $a-f_{0}$ and the glueball from the unstout ensembles have a very low signal-to-noise ratio and are not shown. The results at the smallest gluino mass are preliminary.

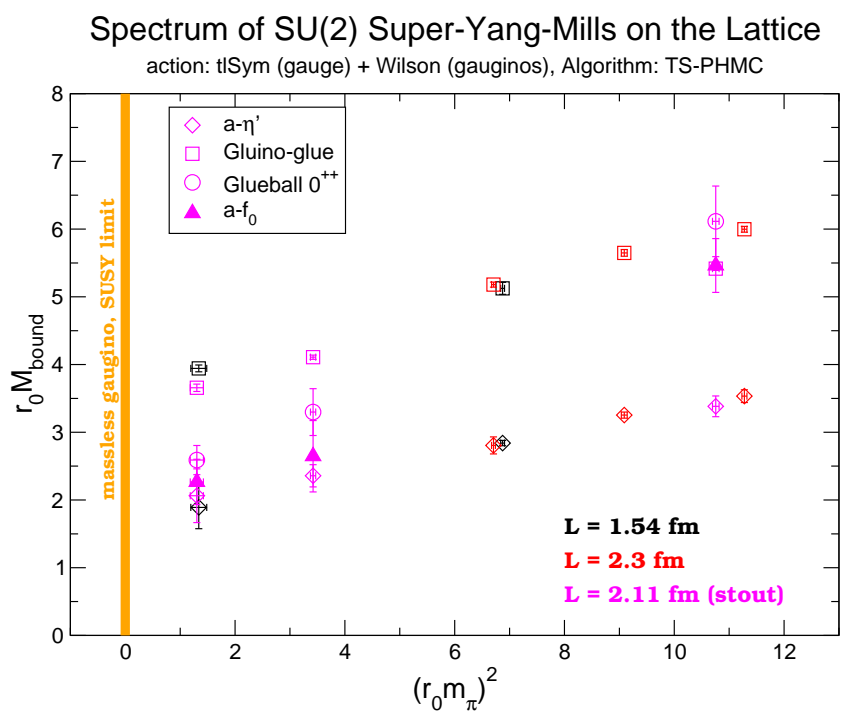

Figure 1: Low-lying bound state masses of $\mathscr{N}=1 \mathrm{SU}(2) \mathrm{SYM}$ as a function of the adjoint pion mass squared (physical units).

The masses in Fig. 1 are multiplied by the Sommer scale parameter and plotted as a function of the squared adjoint pion mass. The lightest simulated adjoint pion mass is about $440 \mathrm{MeV}$ in our units. The vertical line in Fig. 1 highlights the massless gluino limit where SUSY restoration is expected up to $\mathscr{O}(a)$ effects.

The bound state masses appear to be characterised by a linear dependence on $\left(r_{0} m_{\pi}\right)^{2}$. The gluino-glueball ( $\tilde{g} g$ ) with a mass of about $1440 \mathrm{MeV}$ (in our unit where $r_{0}=0.5 \mathrm{fm}$ ) turns out to be considerably heavier than the $a-\eta^{\prime}$ with a mass of $810 \mathrm{MeV}$. Furthermore, the masses of the scalar glueball and the scalar meson $a-f_{0}$ converge to a common point with the pseudoscalar near the region where SUSY is expected. The behaviour of scalars is compatible with mixing between $0^{+}$glueball and $a-f_{0}$. The behaviour of masses suggests a lower supermultiplet, while the spin- $1 / 2$ candidate remains heavier up to the smallest simulated gluino mass in this simulation. Whether this 
outcome is a discretisation artefact or a physical effect, as claimed in [22], should become clear in future studies at finer lattice spacings. As the data at small gluino mass are preliminary, it would be premature to draw conclusions.

\section{Spontaneous symmetry breaking}

An important feature of the strong interaction dynamics of SYM theory is the spontaneous breaking of the discrete chiral symmetry. The appearance of two degenerate ground states in case of SU(2) gauge group was observed in [23] on a small $\left(6^{3} \cdot 12\right)$ lattice by neglecting the sign of the Pfaffian.

Recently we repeated this computation on a larger $\left(16^{4}\right)$ lattice and also taking into account the Pfaffian signs. The observed distribution of the gluino condensate is shown in Fig. 2.

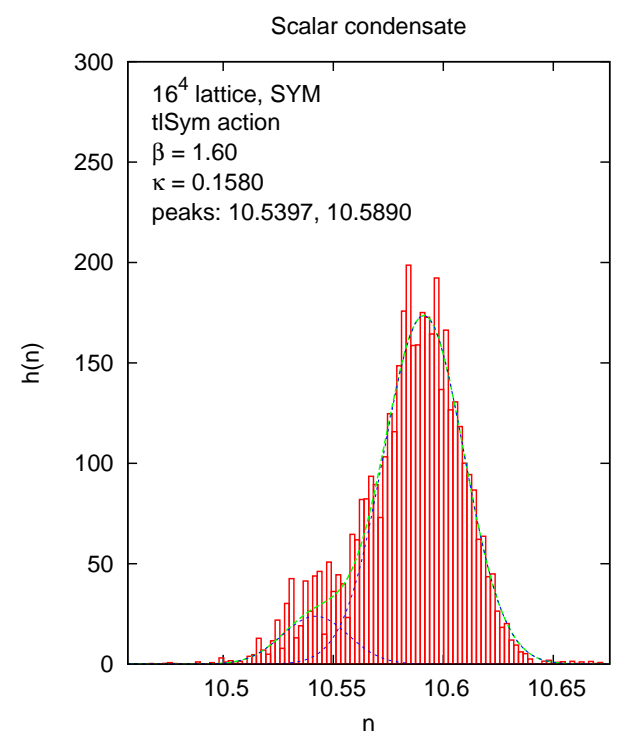

Figure 2: Distribution of the gluino condensate at $\beta=1.6, \kappa=0.158$.

The distribution cannot be fitted with a single Gaussian, but a two-Gaussian fit shown on the figure, corresponding to the two ground states, is reasonable. From the difference of the positions of the two peaks one can, in principle, determine the magnitude of the gluino condensate. This however requires the knowledge of renormalisation constants and therefore is left for a future work.

\section{Conclusion}

In this work first quantitative results of the low-energy spectrum of $\mathscr{N}=1$ supersymmetric Yang-Mills theory are obtained. Physical volumes larger than $2 \mathrm{fm}$ have been simulated, which is the volume usually required for spectrum studies in lattice gauge theory. The comparison of masses on different volumes in otherwise same conditions reveals negligible finite size effects at least for moderate gluino masses. We have collected higher statistics and have used efficient dynamical algorithms such as TS-PHMC, which is suitable for light fermion masses. In addition, 
the supersymmetric Ward identities and other observables like the confinement potential and the gluino condensate have been investigated.

From the results of the mass spectrum the question of the gluino-gluino and gluino-glueball mass splitting remains open. It can only be answered by further simulations allowing an extrapolation to the continuum limit.

\section{References}

[1] G. Veneziano and S. Yankielowicz, Phys. Lett. B 113 (1982) 231.

[2] G.R. Farrar, G. Gabadadze and M. Schwetz, Phys. Rev. D 58 (1998) 015009.

[3] A. Armoni, M. Shifman and G. Veneziano, in From Fields to Strings: Circumnavigating Theoretical Physics, vol. 1, eds. M. Shifman, A. Vainshtein, J. Wheater, World Scientific, Singapore, 2005, p. 353; [hep-th/0403071].

[4] F. Farchioni, G. Münster, T. Sudmann, J. Wuilloud, I. Montvay and E. E. Scholz, PoS(LATTICE 2008) 128, PoS(LATTICE 2007) 135, Eur. Phys. J. C 52 (2007) 305.

[5] G. Curci and G. Veneziano, Nucl. Phys. B 292 (1987) 555.

[6] I. Montvay, Int. J. Mod. Phys. A 17 (2002) 2377.

[7] I. Campos, A. Feo, R. Kirchner, S. Luckmann, I. Montvay, G. Münster, K. Spanderen and J. Westphalen, Eur. Phys. J. C 11 (1999) 507.

[8] A. Donini, M. Guagnelli, P. Hernandez and A. Vladikas, Nucl. Phys. B 523 (1998) 529.

[9] F. Farchioni and R. Peetz, Eur. Phys. J. C 39 (2005) 87.

[10] K. Demmouche, F. Farchioni, A. Ferling, G. Münster, J. Wuilloud, I. Montvay and E. E. Scholz, PoS(Confinement 2008) 136.

[11] K. Demmouche, F. Farchioni, A. Ferling, G. Münster, J. Wuilloud, I. Montvay and E. E. Scholz, PoS(LATTICE 2008) 061.

[12] M.G. Endres, PoS(LATTICE 2008) 025.

[13] J. Giedt, R. Brower, S. Catterall, G. T. Fleming and P. Vranas, Phys. Rev. D 79 (2009) 025015.

[14] G.T. Fleming, J. B. Kogut and P. M. Vranas, Phys. Rev. D 64 (2001) 034510.

[15] C. Morningstar and M.J. Peardon, Phys. Rev. D 69 (2004) 054501.

[16] I. Montvay and E.E. Scholz, Phys. Lett. B 623 (2005) 73.

[17] R. Sommer, Nucl. Phys. B 411 (1994) 839.

[18] M. Albanese et al., Phys. Lett. B 192 (1987) 163.

[19] C. R. Allton et al. [UKQCD Collaboration], Phys. Rev. D 47 (1993) 5128.

[20] M. Lüscher and U. Wolff, Nucl. Phys. B 339 (1990) 222.

[21] F. Farchioni, A. Feo, T. Galla, C. Gebert, R. Kirchner, I. Montvay, G. Münster and A. Vladikas, Eur. Phys. J. C 23 (2002) 719.

[22] L. Bergamin and P. Minkowski, hep-th/0301155.

[23] R. Kirchner, S. Luckmann, I. Montvay, K. Spanderen and J. Westphalen, Phys. Lett. B 446 (1999) 209. 